\title{
Qualitative versus automatic evaluation of CT perfusion parameters in acute posterior circulation ischaemic stroke
}

\author{
Raffaella Capasso ${ }^{1,2}$ (D) Stefano Vallone ${ }^{3} \cdot$ Nicola Serra $^{4} \cdot$ Gabriele Zelent $^{3} \cdot$ Luca Verganti $^{3} \cdot$ Federico Sacchetti $^{3}$. \\ Guido Bigliardi ${ }^{5}$ (D) . Livio Picchetto ${ }^{5}$. Ferdinando Caranci ${ }^{1,2}$ (D) $\cdot$ Andrea Zini ${ }^{6}$ (D)
}

Received: 28 April 2020 / Accepted: 6 August 2020 / Published online: 19 August 2020

(C) The Author(s) 2020

\begin{abstract}
Purpose To compare the diagnostic accuracy (ACC) in the detection of acute posterior circulation strokes between qualitative evaluation of software-generated colour maps and automatic assessment of CT perfusion (CTP) parameters.

Methods Were retrospectively collected 50 patients suspected of acute posterior circulation stroke who underwent to CTP (GE "Lightspeed", 64 slices) within $24 \mathrm{~h}$ after symptom onset between January 2016 and December 2018. The Posterior circulationAcute Stroke Prognosis Early CT Score (pc-ASPECTS) was used for quantifying the extent of ischaemic areas on non-contrast (NC)CT and colour-coded maps generated by CTP4 (GE) and RAPID (iSchemia View) software. Final pc-ASPECTS was calculated on follow-up NCCT and/or MRI (Philips Intera 3.0 T or Philips Achieva Ingenia 1.5 T). RAPID software also elaborated automatic quantitative mismatch maps.

Results By qualitative evaluation of colour-coded maps, MTT-CTP4D and Tmax-RAPID showed the highest sensitivity (SE) ( $88.6 \%$ and $90.9 \%$, respectively) and ACC ( $84 \%$ and $88 \%$, respectively) compared with the other perfusion parameters (CBV, $\mathrm{CBF}$ ). Baseline NCCT and CBF provided by RAPID quantitative perfusion mismatch maps had the lowest SE (29.6\% and 6.8\%, respectively) and ACC (38\% and 18\%, respectively). CBF and Tmax assessment provided by quantitative RAPID perfusion mismatch maps showed significant lower SE and ACC than qualitative evaluation. No significant differences were found between the pc-ASPECTSs assessed on colour-coded MTT and Tmax maps neither between the scores assessed on colourcoded CBV-CTP4D and CBF-RAPID maps.

Conclusion Qualitative analysis of colour-coded maps resulted more sensitive and accurate in the detection of ischaemic changes than automatic quantitative analysis.
\end{abstract}

Keywords CT perfusion $\cdot$ Posterior ischaemic stroke $\cdot$ Perfusion maps $\cdot$ Automatic $\cdot$ RAPID

Raffaella Capasso

dott.ssacapasso@gmail.com

1 Department of Medicine and Health Science "V. Tiberio", University of Molise, Via Francesco De Sanctis, 1, 86100 Campobasso, Italy

2 Department of Precision Medicine, School of Medicine, "Luigi Vanvitelli" University of Campania, Naples, Italy

3 Neuroradiology Unit, Ospedale Civile S.Agostino-Estense, Azienda Ospedaliero-Universitaria di Modena, Dipartimento di Neuroscienze, University of Modena and Reggio Emilia, Modena, Italy

4 Statistic Unit, Department of Public Health, University of Federico II, Naples, Italy

5 Stroke Unit, Ospedale Civile S.Agostino-Estense, Azienda Ospedaliero-Universitaria di Modena, Dipartimento di Neuroscienze, University of Modena and Reggio Emilia, Modena, Italy

6 IRCCS Istituto delle Scienze Neurologiche di Bologna, Department of Neurology and Stroke Center, Maggiore Hospital, Bologna, Italy

\section{Introduction}

Posterior circulation (PC) stroke accounts for 20-25\% of ischaemic strokes and is characterised by a wide range of clinical features that make its clinical diagnosis challenging [1-12]. In emergency setting, dynamic CT perfusion (CTP) has been introduced as a particularly useful technique after noncontrast CT (NCCT) given its easy application, reproducibility of quantitative assessment, prompt availability, low cost and tolerability [13-17]. A mismatch between the irreversibly damaged ischaemic core and the extent of hypoperfused tissue at risk of infarction is an attractive model with which to select ischaemic stroke patients for reperfusion therapies [18-23].

Visual inspection of CTP colour-coded maps can be an effective way to discriminate areas of core infarct and penumbra and may be enough to guide therapeutic choice. It is rapid 
and simple to use but its strengths may be affected by variability in post-processing, broad range of imaging and computational approaches, selection of parametric maps, expertise skills and the generally qualitative nature of such approaches [23].

On the other side, quantitative CTP analysis has reported to be efficient in demonstrating acute ischaemia, distinguishing salvageable from unsalvageable ischaemic tissue and predicting therapeutic outcome, although protocols and guidelines for quantitative thresholds vary [24-33]. Differences in CTP hardware and software can affect quantified analysis and clearly defined thresholds to manage therapeutic approach have yet to be standardised [24-34]. Thus, expert consensus has underlined the need for standardization in the acquisition, processing and analysis of perfusion imaging [23].

In this context, the recent introduction of a fast, vendor- and operator-independent computational tool working by totally automated lesion segmentation and pixel-wise parametric thresholding for semi-quantitation (RApid processing of PerfusIon and Diffusion [RAPID]) seems to overcome the limits of qualitative approaches to CTP imaging in acute stroke [23, 35-37]. This technological advance is reported to have the potential to improve the standardization and reproducibility of interpretation of perfusion imaging [35].

\section{Aim of the study}

The diagnostic value of CTP in the posterior circulation has been little investigated with few evidences of its reliability and accuracy; moreover, the diagnostic effects of RAPID introduction in the evaluation of infratentorial ischaemic changes have not yet been clarified $[3,4,38-40]$. To the best of our knowledge, these are the first reported instances of PC stroke patient selection using RAPID from European countries.

The aim of this study was to examine and compare the diagnostic accuracy (ACC) in the detection of acute posterior circulation strokes between qualitative evaluation of softwaregenerated colour maps [cerebral blood flow (CBF), cerebral blood volume (CBV), mean transit time (MTT) and time-to maximum (Tmax)] and automatic assessment of CTP data by RAPID software.

\section{Methods}

\section{Study population and patient selection}

According to the approval of the local ethical committee (Area Vasta Emilia Nord, AVEN), imaging data were retrospectively collected from a prospective database of 1498 consecutive patients admitted to the stroke unit of our hospital between January 2016 and December 2018. Written informed consent was waived for this study. All patients were initially evaluated by vascular neurologist in the emergency setting with initiation of an institutional stroke protocol facilitating expedited triage, imaging, interpretation and treatment when appropriate. Patients were enrolled regardless of therapy. The inclusion criteria for this study were (1) suspected acute posterior circulation ischaemic stroke as defined in the Oxfordshire classification [41]; (2) multimodal CT scan dataset including NCCT, supra-aortic CT angiography (CTA) and CTP performed on admission; (3) CT scan performed $<24 \mathrm{~h}$ after symptom onset. Exclusion criteria were (1) nondiagnostic image quality, (2) evidence of another cause of neurological deficits (prior stroke with residual deficit, intracranial haemorrhage, tumour etc.), (3) incomplete coverage by the CTP slab of all posterior circulation Acute Stroke Prognosis Early CT Score (pc-ASPECTS) regions and (4) missing follow-up $\mathrm{CT}$ or magnetic resonance imaging (MRI).

Out of all patients who underwent multimodal CT due to suspected acute stroke, were selected control patients who fulfilled the same inclusion and exclusion criteria as our case cohort but did not show an acute posterior circulation ischaemia on follow-up CT/MRI scan neither change in at least two perfusion parameters in the same location.

\section{Imaging protocol}

Patients underwent an institutional stroke imaging protocol including NCCT, CTA and CTP at admission. CT protocol was conducted on a 40-mm, 64-detector row clinical system (Lightspeed VCT, GE Healthcare, Milwaukee, Wisconsin). NCCT helical scans were performed from the skull base to the vertex $(120 \mathrm{kV}, 100-350$ auto-mA, $5 \mathrm{~mm}$ section thickness). CTAs were conducted from the aortic arch to the top of frontal sinus (120 kV; 200-350 auto-mA; section thickness/ interval $0.625 / 0.375 \mathrm{~mm}$; scan start $6 \mathrm{~s}$ after bolus tracking at the level of the ascending aorta) after intravenous administration of $60-70 \mathrm{~mL}$ iodinated contrast medium injected at $4 \mathrm{~mL} /$ $\mathrm{s}$ and followed by $50 \mathrm{~mL}$ saline flush.

CTP scans $(80 \mathrm{kV}, 250 \mathrm{~mA}$ and $0.4 \mathrm{~s}$ rotation time) consisted of a continuous $65 \mathrm{~s}$ acquisition that started $7 \mathrm{~s}$ after administration of $50 \mathrm{~mL}$ of iodinated contrast medium injected into an antecubital vein at $4 \mathrm{~mL} / \mathrm{s}$ and followed by $50 \mathrm{~mL}$ saline flush; 400 images were reconstructed covering $80 \mathrm{~mm}$. CTP coverage was lowered to cover the cerebellum to the occipital lobes, including all three levels of the pcASPECTS.

Follow-up imaging consisted in NCCT. If clinically indicated, MRI was performed either soon after the CTP at the admission or later as follow-up control. MRI exams were achieved using two scanners (Philips Intera 3.0 T and Philips Achieva Ingenia 1.5 T, Philips Medical System, Best, The Netherlands) including diffusion-weighted imaging (DWI) and fluid-attenuated inversion recovery in the transverse plane. 


\section{CTP post-processing}

Raw CTP data were initially processed by a commercially available delay-insensitive deconvolution software [CT Perfusion 4D (CTP4D), GE Healthcare, Waukesha, Milwaukee, Wisconsin]. All patients were obtained CBF, CBV and MTT maps after positioning a region of interest manually in correspondence to a main arterial vessel and a vein. Then, raw CTP data was sent from scanner to a networked computer running fully automated RAPID software (iSchemaView, California) which generated CBF, CBV, MTT, Tmax maps and lesion segmentation and then sent processed images to institutional Picture and Achieving System (PACS). The software also generated a colour perfusion mismatch map for each patient based on the mismatch model Tmax-CBF, providing a comparison between brain regions with substantial reductions in $\mathrm{CBF}(\mathrm{rCBF}<30 \%)$ in pink representing the ischaemic core and regions with significant hypoperfusion in green as reflected by delays in contrast arrival times (Tmax $>6.0 \mathrm{~s}$ ). The difference between these volumes (mismatch volume) as well as the ratio between these volumes (mismatch ratio) was automatically calculated.

\section{NCCT and CTP image analysis}

The neuroimaging data was independently reviewed by two board-certified neuroradiologists (with 10 and 4 years of experience in multimodal CT imaging interpretation) blinded to patient clinical information using the institutional PACS; any discrepancies were resolved through a consensus discussion in a separate session.

For NCCT, early ischaemic changes were assessed; an area was considered ischaemic if there was parenchymal hypoattenuation with or without cortical swelling of the brain $[42,43]$. Areas of cortical swelling without hypoattenuation were not considered ischaemic [43]. The pc-ASPECTS was used for quantifying the extent of ischaemic areas. Pc-ASPECTS allots 10 points for ischaemic changes assessed by visual inspection [23, 44]. Two points are subtracted in the cases of ischaemic change in any part of pons and midbrain, one point for either side of the cerebellum, one for either side of the thalamus and one for either side of the posterior cerebral artery supplied territory $[4,23,44$, 45]. A pc-ASPECTS of 10 indicates absence of ischaemic changes in the posterior circulation while a pc-ASPECTS of 0 indicates presence of ischaemic changes in all pc-ASPECTS territories [38].

For CTP, a qualitative evaluation of perfusion deficit was initially performed on colour-coded CBV, CBF and MTT maps generated by CTP4D software. Regions with colorimetric asymmetries on CTP maps in comparison with nonaffected regions (i.e. contralateral side, different vascular territories) were rated as abnormal. A visible qualitative focal reduction in $\mathrm{CBF}$ or $\mathrm{CBV}$ and a focal increase MTT on colour-coded maps was rated as abnormal and evaluated with pc-ASPECTS. When a perfusion deficit was suspected in the maps, it was compared with the NCCT scan to exclude false positive caused by beam hardening artefacts, chronic infarct or overlapping with liquoral spaces [12].

In the same way, in a separate scoring session, colourcoded CBF and Tmax maps automatically generated by RAPID software were then visually evaluated assessing pcASPECTS for each map. For each patient, the quantitative colour perfusion mismatch map was also evaluated: brain regions with reductions in CBF $(<30 \%)$ appeared in pink suggesting ischaemic core while regions with significant increase of Tmax (> $6.0 \mathrm{~s}$ ) indicating hypoperfusion appeared in green; the abnormal areas identified on perfusion mismatch maps were compared with perfusion changes location on colourcoded maps to assess anatomic consistency in order to exclude artefacts.

PCT images showing no alterations in the same location in at least two visually evaluated perfusion parameter maps neither in automatic maps were considered negative [5].

\section{Follow-up imaging evaluation}

Standard follow-up imaging consisted in NCCT for all patients which was used to determine the presence of an infarct. Final pc-ASPECTS was calculated on follow-up NCCT and/ or on MRI if performed.

Follow-up images were compared with PCT studies for anatomic correlation. The initial NCCT and PCT data was then compared with the follow-up NCCT data or MRI data if available, which was used as criterion standard to calculate sensitivity (SE), specificity (SP) and diagnostic ACC of the abnormalities assessed on NCCT scan and CTP maps for the detection of posterior circulation ischaemic stroke.

\section{Statistical analysis}

For each patient, NCCT and PCT maps showing no ischaemic changes despite follow-up imaging that confirmed the stroke were considered as false negative. PCT maps showing alterations out of the territory of the affected arterial vessel (i.e. contralateral side, anterior circulation) were considered as false positive. NCCT and CTP ischaemic changes were considered true positives if there was an anatomic vascular correspondence to findings on follow-up imaging. NCCT and PCT images showing no alterations in patients whose follow-up imaging did not confirm the stroke were considered as true negative.

Main evaluations were:

- the correspondence between the infarcted area (core) assessed with qualitative evaluation of both colour-coded 
CTP4D maps and RAPID maps and the ischaemic lesion confirmed on direct follow-up CT or MRI;

- the correspondence among the perfusion changes identified with the qualitative analysis of colour-coded CTP4D maps, colour-coded RAPID maps and the lesions spotted in the RAPID software-generated colour perfusion mismatch map;

- the comparison of the respective ASPECTSs evaluated on NCCT, colour-coded CTP4D maps and colour-coded RAPID maps with the final ASPECTSs assessed on direct follow-up CT or MRI.

The statistical analysis was performed by Matlab statistical toolbox version 2008 (MathWorks, Natick, MA, USA) for Windows at 32 bit. SE, SP and ACC for selected parameters were computed with correspondence confidence intervals (CIs) at 95\%. The multiple comparison Cochran's $Q$ tests were used to compare differences of percentages among three or more dependent variables, under the consideration of the null hypothesis that there was no difference between the variables. A value of $p<0.05$ was considered statistically significant. When the Cochran's $Q$ test was positive ( $p$ value < 0.05 ), then a minimum required difference for a significant difference between two proportions was calculated using the minimum required differences method with Bonferroni $p$ value corrected for multiple comparisons according to Sheskin. The McNemar's exact test was used to test the difference between two paired proportions. Additional statistical tests were performed on stroke patient parameters using the Friedman's ANOVA test. Particularly, if Friedman's ANOVA test was positive ( $p$ value $<0.05$ ), a Wilcoxon signed rank post hoc test was performed to individualise significant differences between two diagnostic parameters.

Data are expressed as mean value \pm standard deviation (SD) for continuous variables and as absolute number or percentage for categorical variables.

\section{Results}

\section{Study sample}

Among 69 patients meeting inclusion criteria, a total of 50 patients respected the exclusion criteria. Six out of the 50 patients did not show ischaemic core at follow-up imaging neither alteration of at least two perfusion parameters in the same location and were grouped as negative controls. All patients underwent to follow-up NCCT and 28 of them also underwent DWI-MRI. Mean time from multimodal CT study at admission to follow-up CT study and MRI were, respectively, $34.2 \pm 72.6 \mathrm{~h}$ and $76.6 \pm 98.8 \mathrm{~h}$. Out of the 28 patients who undergone MRI, 14 patients $(50 \%)$ underwent DWI study within $8 \mathrm{~h}$ after multimodal CT study at admission.
Vessel occlusion was located in one of more of the posterior circulation branches involving extracranial vertebral artery in 9 patients, intracranial vertebral artery in 5 patients, basilar artery in 15 patients, posterior cerebral artery in 22 patients and superior cerebellar artery in 7 patients. Inside local stroke protocol multimodal neuroimaging (CT, CTA, CTP or MRI) was used for decision making about inclusion/exclusion criteria for revascularization therapies as intravenous thrombolysis and mechanical thrombectomy. In our study, 26 patients had intravenous thrombolysis, 6 patients underwent to thrombectomy/thromboaspiration, 7 patients had intravenous thrombolysis and underwent to thrombectomy/ thromboaspiration while 5 patients were not treated. Negative controls were not treated.

Table 1 provides an overview of demographic features of the study sample and displays the mean ASPECTS evaluated on NCCT at baseline, colour-coded CTP4D maps, colourcoded RAPID maps, follow-up NCCT and DWI-MRI.

\section{Study sample}

\section{Diagnostic accuracy}

Table 2 displays SE, SP, and ACC relative to baseline NCCT, visually evaluated colour-coded MTT, CBV and CBF maps generated by CTP4D software, visually evaluated colourcoded Tmax and CBF maps generated by RAPID software, automatic quantitative Tmax and CBF parameters spotted in the RAPID software-generated colour perfusion mismatch map, using follow-up CT or MRI as criterion standard.

\section{Diagnostic accuracy}

SE, SP and diagnostic ACC of qualitative evaluation on baseline NCCT, colour-coded CTP4D maps (MTT, CBV and CBF), colour-coded Tmax and CBF RAPID maps (Tmax ${ }^{1}$ and $\mathrm{CBF}^{1}$ ), and automatic quantitative Tmax and $\mathrm{CBF}$ RAPID software-generated perfusion mismatch map ( $\operatorname{Tmax}^{2}$ and $\mathrm{CBF}^{2}$ ), were compared.

The sensitivity of qualitative evaluation of colour-coded MTT map and colour-coded $\operatorname{Tmax}^{1}$ map resulted significantly higher than the other ones (MTT: $88.6 \%, p<0.05$; $\operatorname{Tmax}^{1}$ : $90.9 \%, p<0.05$ ), while NCCT at baseline and $\mathrm{CBF}^{2}$ provided by RAPID quantitative perfusion mismatch maps had the lowest values ( $29.6 \%$ and $6.8 \% p<0.05$, respectively). NCCT at baseline and $\mathrm{CBF}^{2}$ provided by RAPID quantitative perfusion mismatch maps also showed false negative cases in most patients variously located in one or more pc-ASPECTS territories. Automatic $\operatorname{Tmax}^{2}$ evaluation provided by RAPID overlooked 11 ischaemic lesions which mostly (8/11) were lacunar infarcts involving thalamus $(4 / 8)$ or brainstem $(4 / 8)$ while the remaining were larger lesions involving occipital lobe an cerebellum (3/11) (Fig. 1). Visual qualitative 
Table 1 Demographic features and mean ASPECTSs evaluated on respective images of all 50 patients included in the study

\begin{tabular}{|c|c|c|c|c|}
\hline & \multicolumn{2}{|l|}{ Stroke patients } & \multicolumn{2}{|c|}{ Negative controls } \\
\hline & Nr./percentage & Mean $\pm \mathrm{SD}$ & Nr./percentage & Mean $\pm \mathrm{SD}$ \\
\hline Nr. patients & 44 & - & 6 & - \\
\hline Age & - & $71.09 \pm 15.64$ & - & $69 \pm 9.52$ \\
\hline Gender (male) & $(30 / 44) / 68.18 \%$ & - & $(4 / 6) / 66.67 \%$ & - \\
\hline \multirow[t]{2}{*}{ Baseline NCCT } & \multirow[t]{2}{*}{-} & ASPECTS & \multirow[t]{2}{*}{ - } & ASPECTS \\
\hline & & $9.48 \pm 1.12$ & & $10.0 \pm 0.0$ \\
\hline MTT (CT perfusion 4D) & - & $6.80 \pm 2.84$ & - & $9.17 \pm 0.90$ \\
\hline CBV (CT perfusion 4D) & - & $8.16 \pm 2.40$ & - & $10.0 \pm 0.0$ \\
\hline CBF (CT perfusion 4D) & - & $7.52 \pm 2.61$ & - & $10.0 \pm 0.0$ \\
\hline Tmax (colour-coded rapid) & - & $6.41 \pm 3.06$ & - & $9.33 \pm 0.94$ \\
\hline CBF (colour-coded rapid) & - & $8.16 \pm 2.33$ & - & $10.0 \pm 0.0$ \\
\hline follow-up NCCT & - & $6.98 \pm 2.51$ (44 patients) & - & $10.0 \pm 0.0$ \\
\hline Final ASPECTS & - & $6.93 \pm 2.35$ (44 patients) & - & $10.0 \pm 0.0$ \\
\hline MR-DWI & - & $7.04 \pm 2.14(28$ patients $)$ & - & - \\
\hline Follow-up NCCT & - & $6.93 \pm 2.69(16$ patients $)$ & - & $10.0 \pm 0.0$ \\
\hline
\end{tabular}

evaluation of MTT overlooked ischaemic changes in five patients located in pc-ASPECT pons-midbrain region in five cases associated to thalamus involvement in two cases, visual qualitative evaluation of $\operatorname{Tmax}^{1}$ overlooked ischaemic changes in four patients located in pc-ASPECT pons-midbrain regions in three cases and thalamus in one case (Fig. 2). No significant difference was found between MTT map and $\operatorname{Tmax}^{1}$ map sensitivities $(88.6 \%<90.9 \%, p>0.05)$. For $\mathrm{CBV}$ and $\mathrm{CBF}$ parameters, the number of false negative cases located in one or more pc-ASPECTS regions probably reflected the potentially salvageable tissue. However, it is relevant that automatic assessment of $\mathrm{CBF}^{2}$ provided by RAPID mismatch map resulted negative for core extent in most (41/ 44) of patients, among them 20 were lacunar infarcts involving thalamus (15/20) and brainstem (5/20).

About specificity, there was a significant difference among parameters but post hoc test did not confirm significant differences considering all pairwise comparison. However, qualitative
Table 2 Sensitivity, specificity, diagnostic accuracy, false negative and false positive cases relative to baseline NCCT, visually evaluated colour-coded MTT, CBV and CBF maps generated by CT Perfusion 4D software, visually evaluated colourcoded Tmax and CBF maps generated by RAPID software ( $\operatorname{mmax}^{1}$ and $\mathrm{CBF}^{1}$, respectively), automatic quantitative Tmax and CBF parameters spotted in the RAPID software-generated colour perfusion mismatch map ( $\max ^{2}$ and $\mathrm{CBF}^{2}$, respectively)

\begin{tabular}{|c|c|c|c|c|c|}
\hline Parameters & $\begin{array}{l}\text { Sensitivity (CI at } \\
95 \%)\end{array}$ & $\begin{array}{l}\text { Specificity (CI at } \\
95 \%)\end{array}$ & $\begin{array}{l}\text { Accuracy (CI at } \\
95 \% \text { ) }\end{array}$ & $\begin{array}{l}\text { False } \\
\text { negative }\end{array}$ & $\begin{array}{l}\text { False } \\
\text { positive }\end{array}$ \\
\hline Baseline NCCT & $\begin{array}{l}29.6 \%(13 / 44) \\
\quad(16.8-45.2 \%)\end{array}$ & $\begin{array}{l}100 \%(6 / 6) \\
\quad(54.1-100 \%)\end{array}$ & $\begin{array}{l}38 \%(19 / 50) \\
\quad(25-52.7 \%)\end{array}$ & $\begin{array}{l}31 / 44 \\
70.4 \%\end{array}$ & - \\
\hline $\begin{array}{l}\text { MTT (CT } \\
\quad \text { perfusion 4D) }\end{array}$ & $\begin{array}{l}88.6 \%(39 / 44) \\
\quad(75.4-96.2 \%)\end{array}$ & $\begin{array}{l}50 \%(3 / 6) \\
\quad(11.8-88.2 \%)\end{array}$ & $\begin{array}{l}84 \%(42 / 50) \\
\quad(70.3-92.8 \%)\end{array}$ & $\begin{array}{l}5 / 44 \\
11.4 \%\end{array}$ & $\begin{array}{l}3 / 6 \\
50 \%\end{array}$ \\
\hline $\begin{array}{l}\text { CBV (CT } \\
\text { perfusion 4D) }\end{array}$ & $\begin{array}{l}63.6 \%(28 / 44) \\
\quad(47.8-77.6 \%)\end{array}$ & $\begin{array}{l}100 \%(6 / 6) \\
\quad(54.1-100 \%)\end{array}$ & $\begin{array}{l}68 \%(34 / 50) \\
\quad(53.2-80.3 \%)\end{array}$ & $\begin{array}{l}16 / 44 \\
36.4 \%\end{array}$ & - \\
\hline $\begin{array}{l}\mathrm{CBF}(\mathrm{CT} \\
\text { perfusion 4D) }\end{array}$ & $\begin{array}{l}81.8 \%(36 / 44) \\
\quad(67.3-91.8 \%)\end{array}$ & $\begin{array}{l}100 \%(6 / 6) \\
\quad(54.1-100 \%)\end{array}$ & $\begin{array}{l}84 \%(42 / 50) \\
\quad(70.3-92.8 \%)\end{array}$ & $\begin{array}{l}8 / 44 \\
18.2 \%\end{array}$ & - \\
\hline $\begin{array}{l}\text { Tmax }{ }^{1} \\
\quad \text { (colour-coded } \\
\text { rapid) }\end{array}$ & $\begin{array}{l}90.9 \%(40 / 44) \\
\quad(78.3-97.5 \%)\end{array}$ & $\begin{array}{l}66.7 \%(4 / 6) \\
\quad(22.3-95.7 \%)\end{array}$ & $\begin{array}{l}88 \%(44 / 50) \\
\quad(75-95.6 \%)\end{array}$ & $\begin{array}{l}4 / 44 \\
9.1 \%\end{array}$ & $\begin{array}{l}2 / 6 \\
33.3 \%\end{array}$ \\
\hline $\begin{array}{l}\mathrm{CBF}^{1} \\
\quad \text { (colour-coded } \\
\text { rapid) }\end{array}$ & $\begin{array}{l}61.4 \%(27 / 44) \\
\quad(45.5-75.6 \%)\end{array}$ & $\begin{array}{l}100 \%(6 / 6) \\
\quad(54.1-100 \%)\end{array}$ & $\begin{array}{l}66 \%(33 / 50) \\
\quad(51.1-78.6 \%)\end{array}$ & $\begin{array}{l}17 / 44 \\
38.6 \%\end{array}$ & - \\
\hline $\begin{array}{l}\text { Tmax } \\
\text { (quantitative } \\
\text { rapid) }\end{array}$ & $\begin{array}{l}65.9 \%(29 / 44) \\
\quad(50.1-79.5 \%)\end{array}$ & $\begin{array}{l}100 \%(6 / 6) \\
\quad(54.1-100 \%)\end{array}$ & $\begin{array}{l}70 \%(35 / 50) \\
\quad(55.2-81.9 \%)\end{array}$ & $\begin{array}{l}11 / 44 \\
25 \%\end{array}$ & $\begin{array}{l}4 / 6 \\
66.7 \%\end{array}$ \\
\hline $\begin{array}{l}\mathrm{CBF}^{2} \text { (quantitative } \\
\text { Rapid) }\end{array}$ & $\begin{array}{l}6.8 \%(3 / 44) \\
\quad(1.4-18.7 \%)\end{array}$ & $\begin{array}{l}100 \%(6 / 6) \\
\quad(54.1-100 \%)\end{array}$ & $\begin{array}{l}18 \%(9 / 50) \\
\quad(9.1-31.5 \%)\end{array}$ & $\begin{array}{l}41 / 44 \\
93.2 \%\end{array}$ & - \\
\hline
\end{tabular}

For each patient, follow-up CT or MRI was used as criterion standard. Confidence interval (CI) at 95\% 
Fig. 1 Left thalamic lacunar infarct: a ASPECTS 10 on initial NCCT, b RAPID colour-coded Tmax map shows perfusion alteration at left thalamic level but not displayed on RAPID automatic mismatch map (c); the lesion was confirmed on DWI (d) and also visible on follow-up CT (e)
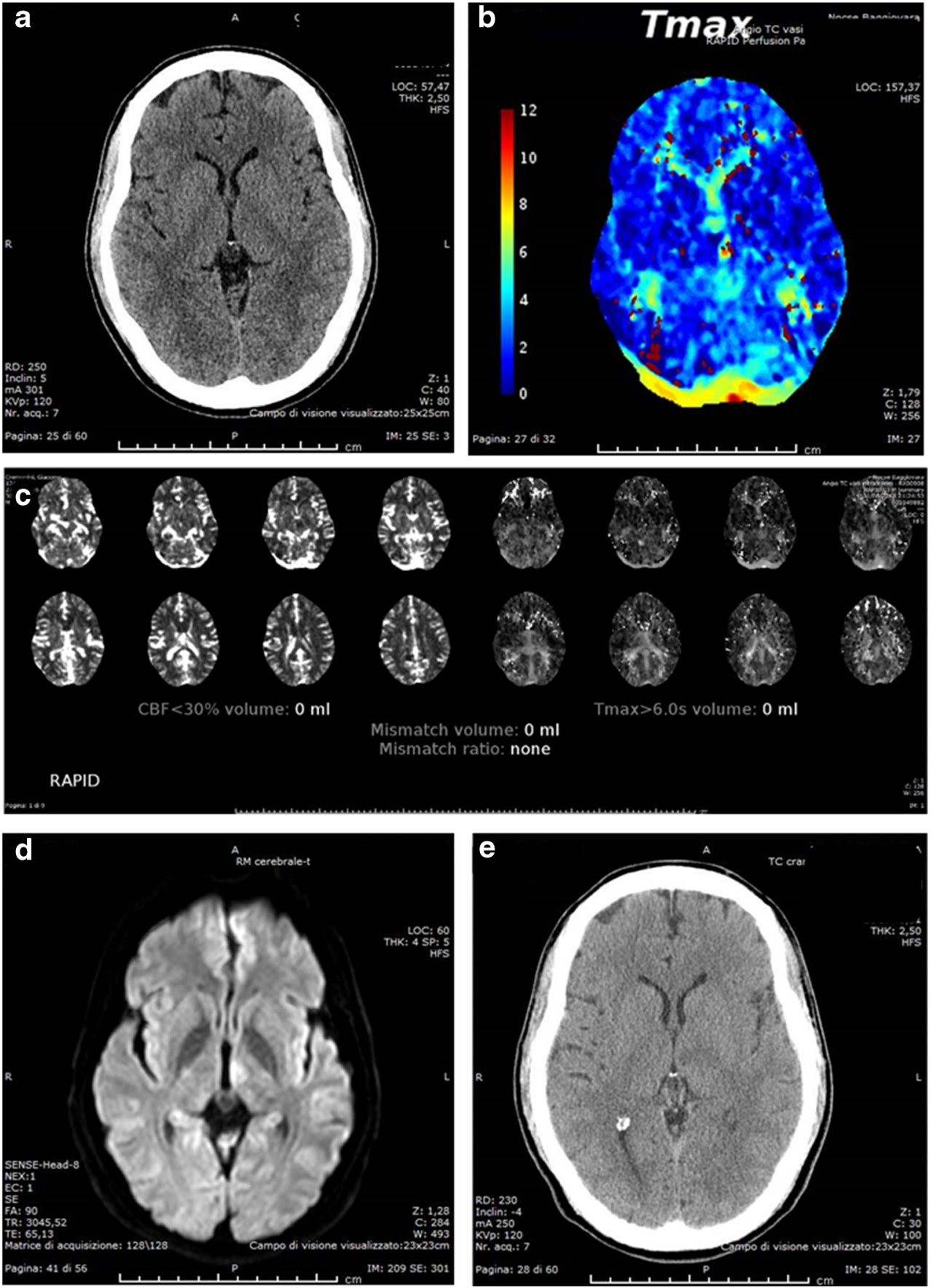

evaluation of MTT and Tmax ${ }^{1}$ maps and automatic evaluation of $\mathrm{Tmax}^{2}$ provided by RAPID quantitative perfusion mismatch maps were characterised by some false positive cases showing perfusion disturbance in anterior circulation territory.

About diagnostic accuracy, MTT and $\operatorname{Tmax}^{1}$ showed the highest values ( $84 \%$ and $88 \% p<0.05$, respectively) while CT at baseline and $\mathrm{CBF}^{2}$ provided by RAPID quantitative perfusion mismatch maps had the lowest values (38\% and $18 \%$ $p<0.05$, respectively). Between MTT and Tmax ${ }^{1}$ map, there was no significant difference $(88 \%>84 \%, p>0.05)$ in terms of diagnostic accuracy.
In addition, sensitivity, specificity and diagnostic accuracy were compared among $\mathrm{CBF}, \mathrm{CBF}^{1}$ and $\mathrm{CBF}^{2}$ parameters (respectively, qualitatively evaluated on colour-coded CTP4D, colour-coded RAPID maps and automatically provided by RAPID quantitative perfusion mismatch maps) and between $\operatorname{Tmax}^{1}$ and $\operatorname{Tmax}^{2}$ parameters (respectively, qualitatively evaluated on colour-coded CTP4D and automatically provided by RAPID quantitative perfusion mismatch maps) (Table 3).

$\mathrm{CBF}^{2}$ assessment provided by quantitative RAPID perfusion mismatch maps showed significant lowest sensitivity 

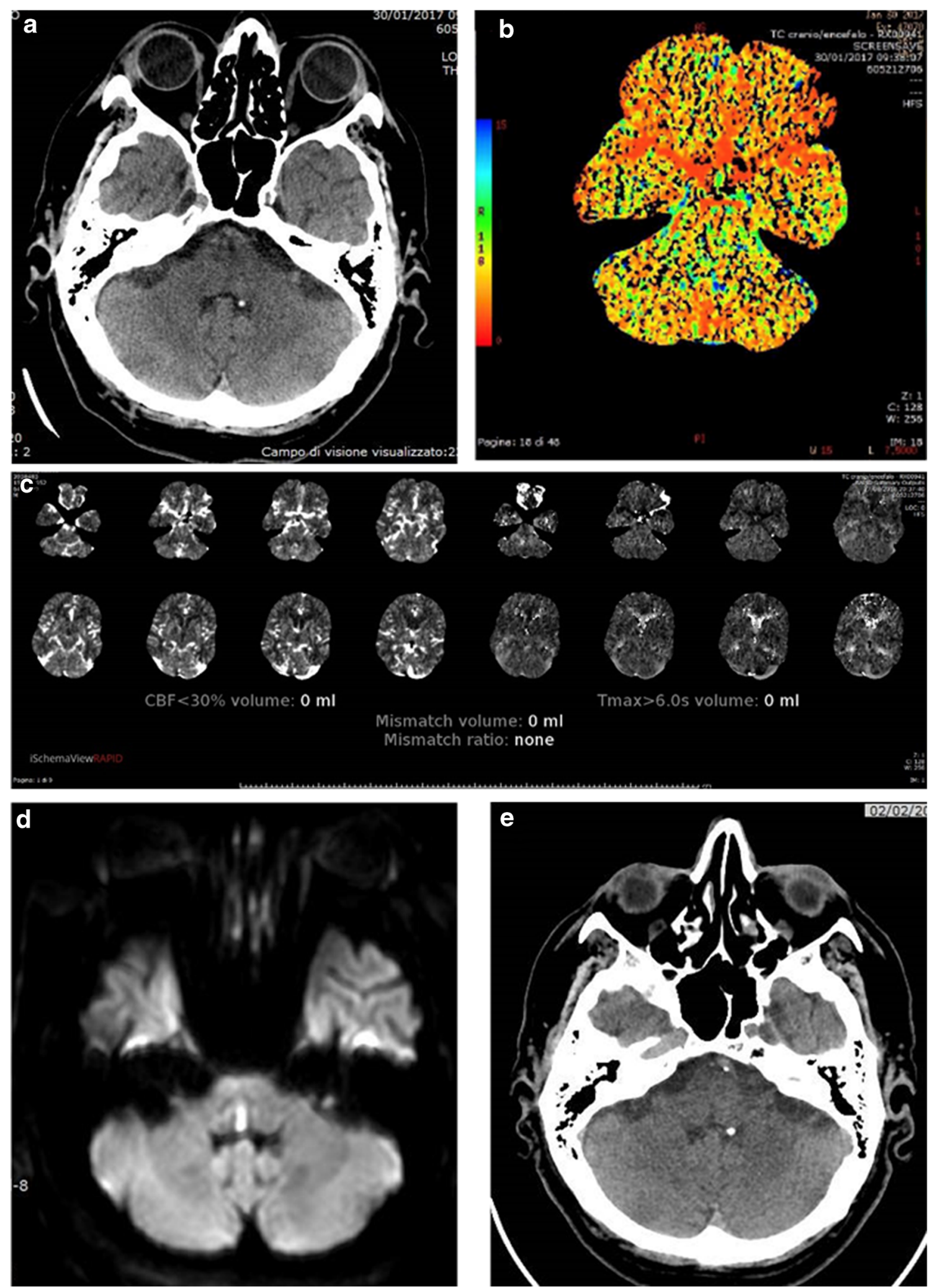

Fig. 2 Mesencephalic lacunar infarct not clearly identified on initial NCCT (a), neither on colour-coded MTT (b) map and RAPID automatic mismatch map (c) but detected by DWI (d) and confirmed on follow-up CT (e)

(6.8\%) in comparison with qualitative evaluations of both colour-coded CBF-CTP4D and CBF $^{\mathbf{1}}$-RAPID maps (81.8\% and $61.4 \%$, respectively); no significant difference of sensitivity was found between qualitative evaluations of colour-coded CBF-CTP4D and CBF $^{1}$-RAPID maps $(81.8 \%>61.4 \%, p>0.05)$. Analogous results were observed 
Table 3 Comparison among CBF parameters and Tmax parameters evaluated in this study in terms of sensitivity, specificity and diagnostic accuracy

\begin{tabular}{|c|c|c|c|c|}
\hline Parameters & $\begin{array}{l}\text { CBF (colour-coded CT } \\
\text { perfusion 4D) }\end{array}$ & $\begin{array}{l}\mathrm{CBF}^{1} \text { (colour- } \\
\text { coded rapid) }\end{array}$ & $\begin{array}{l}\mathrm{CBF}^{2} \text { (quantitative } \\
\text { RAPID) }\end{array}$ & Statistical test \\
\hline Sensitivity & $81.8 \%(36 / 44)$ & $61.4 \%(27 / 44)$ & $6.8 \%(3 / 44)$ & $p<0.001(\mathrm{Q})$ \\
\hline Specificity & $100 \%(6 / 6)$ & $100 \%(6 / 6)$ & $100 \%(6 / 6)$ & $p>0.05(\mathrm{Q})$ \\
\hline $\begin{array}{l}\text { Diagnostic } \\
\text { Accuracy }\end{array}$ & $84 \%(42 / 50)$ & $66 \%(33 / 50)$ & $18 \%(9 / 50)$ & $p<0.001(\mathrm{Q})$ \\
\hline Parameters & & $\begin{array}{l}\operatorname{Tmax}^{1} \\
\quad \text { (colour-coded } \\
\text { rapid) }\end{array}$ & $\begin{array}{l}\operatorname{Tmax}^{2} \\
\text { (quantitative } \\
\text { RAPID) }\end{array}$ & Statistical test \\
\hline Sensitivity & & $90.9 \%(40 / 44)$ & $65.9 \%(29 / 44)$ & $\begin{array}{r}90.9 \%>65.9 \% \\
p=0.001(\mathrm{M})\end{array}$ \\
\hline Specificity & & $69.7 \%(4 / 6)$ & $100 \%(6 / 6)$ & $\begin{array}{c}69.7 .9 \%<100 \%, \\
p=0.5(\mathrm{M})\end{array}$ \\
\hline $\begin{array}{l}\text { Diagnostic } \\
\text { Accuracy }\end{array}$ & & $88 \%(44 / 50)$ & $70 \%(35 / 50)$ & $\begin{array}{l}88 \%>70 \% \\
\quad p=0.0225(\mathrm{M})\end{array}$ \\
\hline
\end{tabular}

$Q$ Cochran's $Q$ test, $M$ McNemar exact test for diagnostic accuracy, while there were no significant differences of specificity among $\mathrm{CBF}, \mathrm{CBF}^{1}$ and $\mathrm{CBF}^{2}$ parameters (respectively, qualitatively evaluated on colour-coded CTP4D and RAPID maps and automatically provided by RAPID quantitative perfusion mismatch maps).

Qualitative evaluation of colour-coded Tmax ${ }^{1}$-RAPID maps showed significant higher sensitivity and accuracy than quantitative assessment of $\operatorname{Tmax}^{2}$ automatically provided by RAPID perfusion mismatch maps $(90.9 \%>65.9 \%$ and $88 \%>70 \%$, respectively) while about specificity, there were no significant difference $(69.7 .9 \%<100 \%, p=0.5)$.

\section{Comparison between qualitative and automatic quantitative perfusion parameter evaluations}

\section{PC-ASPECTS}

Interobserver agreement for visual evaluation and ASPECT scoring of MTT, CBV, CBF, Tmax ${ }^{1}$ and $\mathrm{CBF}^{1}$ maps was established by the exact count approach: MTT, 0.82; CBV, 0.88; CBF, 0.86; Tmax $^{1}, 0.84 ; \mathrm{CBF}^{1}, 0.8$.

Pc-ASPECTSs assessed for each qualitative imaging evaluation [NCCT at baseline, visually evaluated colour-coded MTT, CBV and CBF maps generated by CTP4D software, visually evaluated colour-coded $\operatorname{Tmax}^{1}$ and $\mathrm{CBF}^{1}$ maps generated by RAPID software and the reference standard imaging (follow-up NCCT or MRI)] in stroke patients were analysed by the Friedman's ANOVA test and pairwise Wilcoxon signed-rank post hoc test (Table 4). No significant differences were revealed among values corresponding to the reference standard (2.82), MTT (colour-coded CTP4D, 2.80) and Tmax $^{1}$ (colour-coded Rapid, 2.48) neither between MTT (colour-coded CTP4D) and Tmax ${ }^{1}$ (colour-coded Rapid). No significant differences were even found between CBV (colour-coded CTP4D, 5.14) and $\mathrm{CBF}^{1}$ (colour-coded Rapid, 4.90) values. NCCT at baseline, instead, significantly differed from all the other parameters.

\section{Pairwise comparison}

\section{Discussion}

\section{Ischaemic lesion detection}

Brain imaging plays a key role in the evaluation of patients suspected of ischaemic stroke allowing to reach a confident diagnosis of infratentorial stroke $[5,12]$. MRI with DWI represents the diagnostic gold standard for the detection of ischaemic lesions in the PC territories [4, 5]. However, in many institutions, CT is more readily accessible in the acute phase and less time consuming than MRI being also helpful if MRI is contraindicated or unavailable $[1,4,5]$. CTP has gained widespread application thanks to its accessibility, proved higher sensitivity and accuracy than NCCT alone in detecting acute ischaemic changes also in PC territories [3, 5, 12, 17].

While the infarcted core typically shows a uniform decrease in $\mathrm{CBF}$ and $\mathrm{CBV}$, the penumbra is characterised by a reduction of the $\mathrm{CBF}$ value with a normal or even increased CBV due to the vasodilation of the precapillary arteries and venous obstruction [12,19]. Such areas can also be characterised by prolonged MTT extending beyond areas of core infarct and have been called CBV/MTT mismatch [20]. According to recent evidence, relative cerebral blood flow (rCBF) seems to better perform than other parameters, including $\mathrm{CBV}$, in predicting the infarct core, and Tmax is reported to more accurately measure the penumbra in patients with acute ischaemic stroke: CBF/Tmax mismatch [18, 19, 23]. 
Table 4 Pairwise comparison with Wilcoxon test after positive Friedman's ANOVA test; for each tested variable (left column), the other variables from which (right column) it significantly differed are reported

\begin{tabular}{|c|c|c|}
\hline Variables & $\begin{array}{l}\text { Mean } \\
\text { rank }\end{array}$ & Significant differences $(p<0.05)$ \\
\hline $\begin{array}{l}\text { (1) Reference standard } \\
\text { imaging }\end{array}$ & 2.82 & $\begin{array}{l}\text { NCCT at baseline, CBV (colour-coded CT Perfusion 4D), CBF } \\
\text { (colour-coded CT Perfusion 4D), } \mathrm{CBF}^{1} \text { (colour-coded rapid) }\end{array}$ \\
\hline (2) NCCT at baseline & 6.08 & All other variables \\
\hline $\begin{array}{l}\text { (3) MTT (colour-coded CT } \\
\text { perfusion 4D) }\end{array}$ & 2.80 & $\begin{array}{l}\text { NCCT at baseline, CBV (colour-coded CT perfusion 4D), CBF } \\
\text { (colour-coded CT perfusion } 4 \mathrm{D}), \mathrm{CBF}^{1} \text { (colour-coded rapid) }\end{array}$ \\
\hline $\begin{array}{l}\text { (4) } \mathrm{CBV} \text { (colour-coded CT } \\
\text { perfusion } 4 \mathrm{D} \text { ) }\end{array}$ & 5.14 & $\begin{array}{l}\text { Reference standard imaging, NCCT at baseline, MTT (colour-coded } \\
\text { CT perfusion 4D), CBF (colour-coded CT perfusion 4D), Tmax } \\
\text { (colour-coded rapid) }\end{array}$ \\
\hline $\begin{array}{l}\text { (5) } \mathrm{CBF} \text { (colour-coded CT } \\
\text { perfusion } 4 \mathrm{D} \text { ) }\end{array}$ & 3.80 & All other variables \\
\hline $\begin{array}{l}\text { (6) } \operatorname{Tmax}^{1} \text { (colour-coded } \\
\text { rapid) }\end{array}$ & 2.48 & $\begin{array}{l}\text { NCCT at baseline, CBV (colour-coded CT Perfusion 4D), CBF } \\
\text { (colour-coded CT Perfusion 4D), } \text { CBF}^{1} \text { (colour-coded rapid) }\end{array}$ \\
\hline $\begin{array}{l}\text { (7) } \mathrm{CBF}^{1} \text { (colour-coded } \\
\text { rapid) }\end{array}$ & 4.90 & $\begin{array}{l}\text { Reference standard imaging, NCCT at baseline, MTT (colour-coded } \\
\text { CT perfusion 4D), CBF (colour-coded CT perfusion 4D), Tmax }{ }^{1} \\
\text { (colour-coded rapid) }\end{array}$ \\
\hline
\end{tabular}

Furthermore, ASPECTSs assessed by qualitative evaluations were compared with the final score assigned by the reference standard imaging (control NCCT or MRI), distinguishing if the qualitative score was equal, higher and lower than the reference standard one. The comparison is resumed in Table 5.

Baseline NCCT showed significant most frequent higher values $(84 \%, p<0.05)$ and less frequent equal values $(16 \%, p<0.05)$. MTT (colour-coded CT perfusion 4D) and $\operatorname{Tmax}^{1}$ (colour-coded rapid) were the parameters with significant less frequent higher values $(24 \%, p<0.05 ; 22 \%, p<0.05$, respectively) and more frequent lower values (32\%, and $38 \% p<0.05$, respectively) in comparison with the others; MTT (colour-coded CTP4D) and $\operatorname{Tmax}^{1}$ (colour-coded rapid) parameters resulted statistically equivalent to individualise perfusion changes.
Prior studies have shown that ischaemic core presents $>70 \%$ reduction in $\mathrm{CBF}(\mathrm{rCBF}<0.3)$ in comparison with the mean CBF of normally perfused brain parenchyma while a Tmax delay of $>6 \mathrm{~s}$ is a good predictor of critically hypoperfused tissue that is at risk to progress to infarction in the absence of timely reperfusion; RAPID mismatch maps in our study were automatically elaborated according to these thresholds [18, 23, 28-33].

Concordant to other studies, our findings confirmed that CTP in PC allows to detect significantly more ischaemic strokes than NCCT $[4,5,39]$. As NCCT presented low SE and $\mathrm{ACC}$ values, the addiction of CTP in the diagnostic work- up in our patients suspected of an ischaemic posterior circulation stroke significantly increased diagnostic ACC.

\section{MTT and Tmax}

Perfusion change in the posterior circulation has previously been described to be most frequent and pronounced on MTT and Tmax maps [4, 5, 38]. In agreement with other studies, MTT and Tmax visually assessed on colour-coded maps (respectively, generated by CTP4D and RAPID software) in our study were the most significant sensitive and accurate
Table 5 Comparison among the ASPECTSs assessed by qualitative evaluations with the final score assigned by the reference standard imaging

\begin{tabular}{llll}
\hline Parameters & $\%$ higher & $\%$ lower & $\%$ equal \\
\hline NCCT at baseline & $84 \%(42 / 50)$ & $0.0 \%(0 / 50)$ & $16 \%(8 / 50)$ \\
MTT (colour-coded CT perfusion 4D) & $24 \%(12 / 50)$ & $32 \%(16 / 50)$ & $44 \%(22 / 50)$ \\
CBV (colour-coded CT perfusion 4D) & $68 \%(34 / 50)$ & $10 \%(5 / 50)$ & $22 \%(11 / 50)$ \\
CBF (colour-coded CT perfusion 4D) & $42 \%(21 / 50)$ & $14 \%(7 / 50)$ & $44 \%(22 / 50)$ \\
CBF $^{1}$ colour-coded rapid) & $60 \%(30 / 50)$ & $8 \%(4 / 50)$ & $32 \%(16 / 50)$ \\
Tmax $^{1}$ (colour-coded rapid) & $22 \%(11 / 50)$ & $38 \%(19 / 50)$ & $40 \%(20 / 50)$ \\
Statistical test & $p<0.001(\mathrm{Q})$ & $p<0.001(\mathrm{Q})$ & $p<0.001(\mathrm{Q})$ \\
& NCCT, $p<0.05(\mathrm{Sh})$ & $\mathrm{MTT}, p<0.05(\mathrm{Sh})$ & $\mathrm{NCCT}, p<0.05(\mathrm{Sh})$ \\
& $\mathrm{MTT}, p<0.05(\mathrm{Sh})$ & $\mathrm{Tmax}{ }^{1}, p<0.05(\mathrm{Sh})$ & \\
& $\mathrm{Tmax}{ }^{1}, p<0.05(\mathrm{Sh})$ & & \\
\hline
\end{tabular}

$Q$ Cochran's $Q$ test, Sh Sheskin's procedure

Pc-ASPCETS comparison with the final score evaluated on the reference standard imaging 
parameters in the detection of PC ischaemic lesions [5]; furthermore, they showed no significant differences in terms of sensitivity $(88.6 \%<90.9 \%, p>0.05)$ and ACC $(88 \%>84 \%$, $p>0.05)$ despite a superiority of Tmax as described in literature [29].

Automatic quantitative assessment of $\operatorname{Tmax}^{2}$ (provided by RAPID perfusion mismatch maps) presented significant lower sensitivity and accuracy compared with qualitative visual evaluation of $\operatorname{Tmax}^{1}$ maps. Automatic $\operatorname{Tmax}^{2}$ evaluation, qualitative evaluation of MTT and Tmax ${ }^{1}$ overlooked ischaemic changes mainly located in pons-midbrain regions and thalamus.

Despite potential shortcomings due to beam hardening artefacts, previous studies investigating the specificity of CTP in the detection of posterior circulation infarcts demonstrated specificity higher than $90 \%[5,17]$. In our study, CTP parameters presented different specificity values (ranging from 50\% up to $100 \%$ ) but post hoc test revealed no significant differences of specificity among all assessed CTP parameters, and this result was probably due to small number of control patients considered in this study. As expected, CBF resulted more specific than MTT for stroke because MTT values can be prolonged in transitory ischaemic attack as well as stroke [19]. As previously described in literature, we found that qualitatively evaluated MTT and $\operatorname{Tmax}^{1}$ had low specificity for perfusion deficits [12].

\section{CBV and CBF}

The detection of the ischaemic core is most relevant for the prediction of the functional outcome of stroke patients and core extent is described to be better delineated on CBF compared with CBV maps because CBV parameter may underestimate core extent [21,38].

In agreement with these evidences, in our study, qualitative evaluation of CTP4D maps showed lower sensitivity and accuracy of CBV parameter compared with $\mathrm{CBF}$ ones although without significant difference $(63.6<81.8 \%, p>0.05)$.

CTP maps, especially CBF abnormalities, are described to significantly differ among commercial software even when using identical source data [34]; however, we found that visually evaluated $\mathrm{CBF}$ and $\mathrm{CBF}^{1}$ maps - respectively, generated both by CTP4D and RAPID software - presented no significant difference of sensitivity $(81.8 \%>61.4 \%, p>0.05)$ and accuracy $(6.8 \%<18 \%, p>0.05)$. Instead, the automatic quantitative assessment of $\mathrm{CBF}^{2}$ (provided by RAPID perfusion mismatch maps) presented significant lower sensitivity and accuracy as compared with qualitative visual evaluation of both CTP4D and RAPID colour-coded CBF maps. CBV, $\mathrm{CBF}, \mathrm{CBF}^{1}$ and $\mathrm{CBF}^{2}$ parameters presented the highest specificity among all assessed CTP parameters without falsepositive cases; this is a critical point to not erroneously exclude patients from therapeutic intervention.
Despite other reports using the mismatch model have suggested that visual assessment is unreliable and that automatic processing of CTP can provide an operator-independent mismatch classification improving standardization and reproducibility of interpretation (Fig. 3), in our study, RAPID perfusion mismatch maps failed to adequately reveal the ischaemic core extent in the major part of the patients (41/44) as compared with the qualitative analysis [27]. A relevant result of our study was that $\mathrm{CBF}^{2}$ evaluation ( $\mathrm{rCBF}$ automatically provided by RAPID quantitative perfusion mismatch map) was characterised by significant low sensitivity and accuracy values resulting less adequate even than NCCT for detecting ischaemic lesions in PC regions. Indeed, automatic RAPID assessment did not identify ischaemic lesions visually recognised on NCCT in 10 patients (thalamic lacunar infarct in 5 patients and small cerebellar infarct in 3 patients) (Fig. 4). Only in one patient, automatic RAPID mismatch map depicted an ischaemic core (in the left cerebellar hemisphere) not identified on NCCT.

\section{PC-ASPECTS}

The determination of pc-ASPECTs on colour-coded perfusion maps does not require any volumetric software and can be applied to NCCT as well to colour-coded perfusion maps [45].

In our study, the pc-ASPECTSs assessed by NCCT were mainly higher and significantly different from the corresponding final pc-ASPECTs evaluated on control CT/MRI scan and NCCT presented the lowest rate of scores equal to the final ones. These findings are clearly related to the low SE of NCCT which can depict only the ischaemic core and not perfusion changes at risk of infarction.

We found no significant differences among the scores assessed on control CT/MRI scan, colour-coded MTT and $\mathrm{Tmax}^{1}$ maps resulting MTT and $\operatorname{Tmax}^{1}$ the most efficient parameters to evaluate the patients' status. This finding can be interpreted as the result of the progression of ischaemic hypoperfusion to necrosis in most of our patients. Moreover, MTT and $\operatorname{Tmax}^{1}$ maps led to assign lower ASPECTS significantly more $(32 \%, p<0.05 ; 38 \%, p<0.05$, respectively) and higher ASPECTS significantly less $(24 \%, p<0.05 ; 22 \%$, $p<0.05$, respectively) frequently than the other parameters; a lower score than final one reflected the presence of hypoperfusion not progressed to necrosis while a higher score than final one can be interpreted as a worsening of ischaemic damage and/or as an overlooked ischaemic change. In four patients with midbrain infarct, all perfusion colour-coded maps were scored 10 and the automatic quantitative assessment did not identify these lesions.

Interesting was that no significant differences were found between the scores assessed on colour-coded MTT and Tmax ${ }^{1}$ maps neither between the scores assessed on colour-coded 

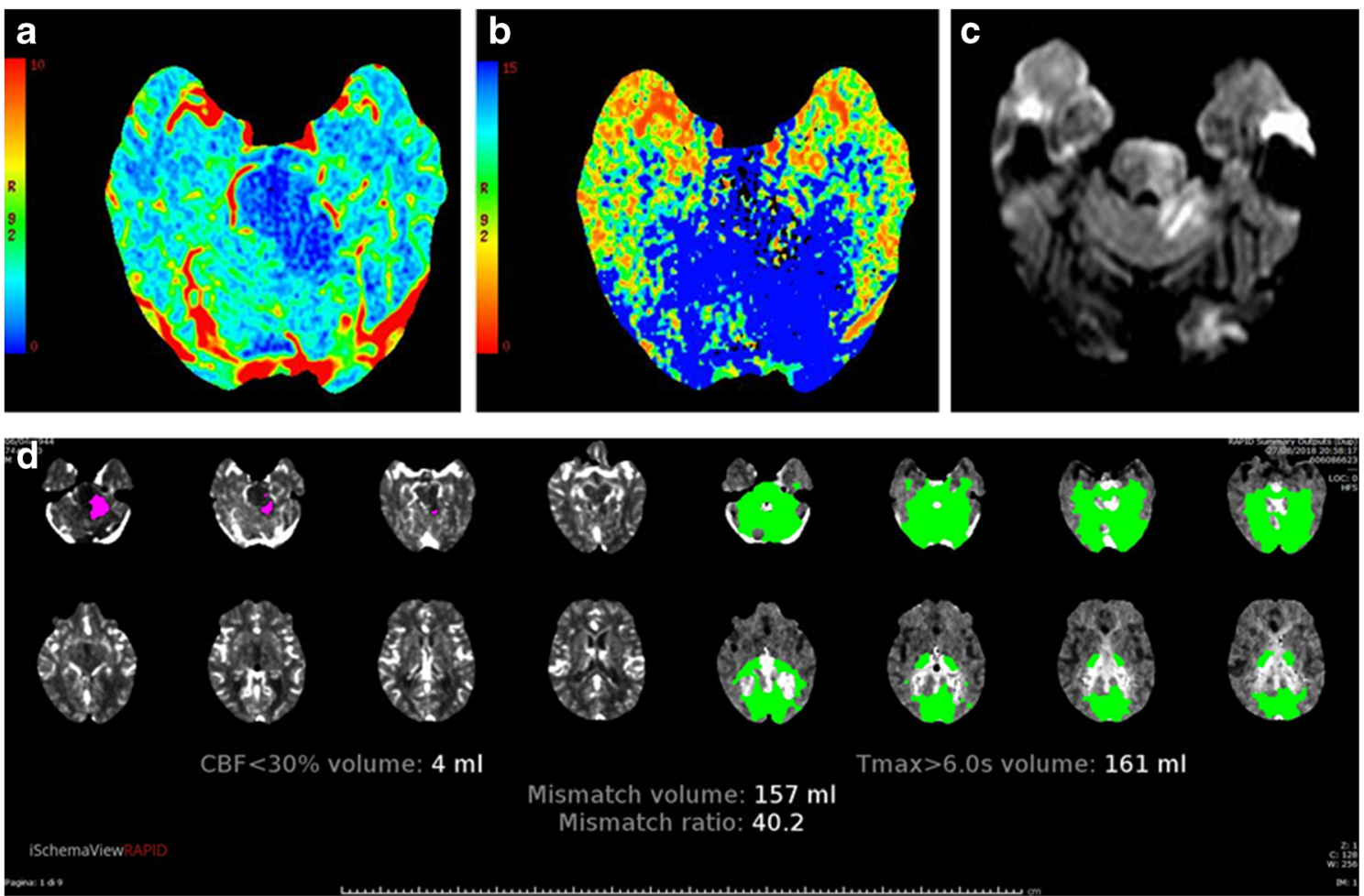

Fig. 3 Left cerebellar and mesencephalic ischaemic changes recognised as core and penumbra, respectively, on CBV (a) and MTT (b) colourcoded CT perfusion 4D maps; the presence of ischaemic core was

$\mathrm{CBV}$ and $\mathrm{CBF}^{1}$ maps; according to this, in our study, the mismatch models MTT-CBV and Tmax ${ }^{1}$-CBF could be considered comparable being not different the determination of hypoperfusion by MTT and $\operatorname{Tmax}^{1}$ neither different the assessment of core extent by $\mathrm{CBV}$ and $\mathrm{CBF}^{1}$.

\section{Limits}

A possible limitation of this study is the retrospective design. Intravenous thrombolysis and thrombectomy may also have influenced SE and SP of perfusion maps because they can lead to temporary reperfusion in the potentially salvageable penumbra. Furthermore, the use of 64-slice CT did not allow the study of the whole parenchyma and a careful positioning of the slab was necessary although resulting not always adequate. This could have influenced the SE of CTP maps.

\section{Conclusion}

In this study, CTP showed good diagnostic accuracy in the identification of acute vascular ischaemic lesions of the PC and the infarct detection of CTP was significantly higher than NCCT $[12,39]$. The most sensitive perfusion parameters were MTT and Tmax. However, sensitivity increases with infarct size being the draw-back of CTP in detection of small-volume confirmed by DWI-MRI performed immediately after to better evaluate the involvement of mesencephalon; the automatic RAPID mismatch map (d) accurately depicted the extent of ischaemic core and penumbra

infarctions [5]. Indeed, detection of ischaemic lesion in the brainstem remains challenging due to beam hardening artefacts $[5,45]$.

Independently to the software employed, qualitative analysis of colour-coded maps resulted more sensitive in the detection of ischaemic changes than automatic quantitative analysis. RAPID software-generated mismatch maps overlooked and underestimated the extent of the ischaemic core in the major part of the patients as compared with the qualitative analysis [12]. In our study, the limits of identification of the lesions by automatic quantitative mismatch maps mainly lied in the thalamus and brainstem. Visual assessment of CTP pcASPECTS on colour-coded perfusion maps revealed comparable scores between MTT and $\operatorname{Tmax}^{1}$ as well as between $\mathrm{CBV}$ and $\mathrm{CBF}^{1}$ suggesting the equivalence of both mismatch models (MTT-CBV and Tmax-CBF) commonly applied in acute setting with implications for treatment decision-making.

For these reasons, the multimodal evaluation of the whole neuroradiological examination (NCCT, all qualitative and quantitative CTP maps) must be underlined [46].

Given the advantages of a more rapid and operator independence elaboration of perfusion maps and their easier interpretation, this opens the potential for automatic software implementation and optimization of perfusion parameters' thresholds for the evaluation of posterior circulation ischaemia $[12,35]$. 

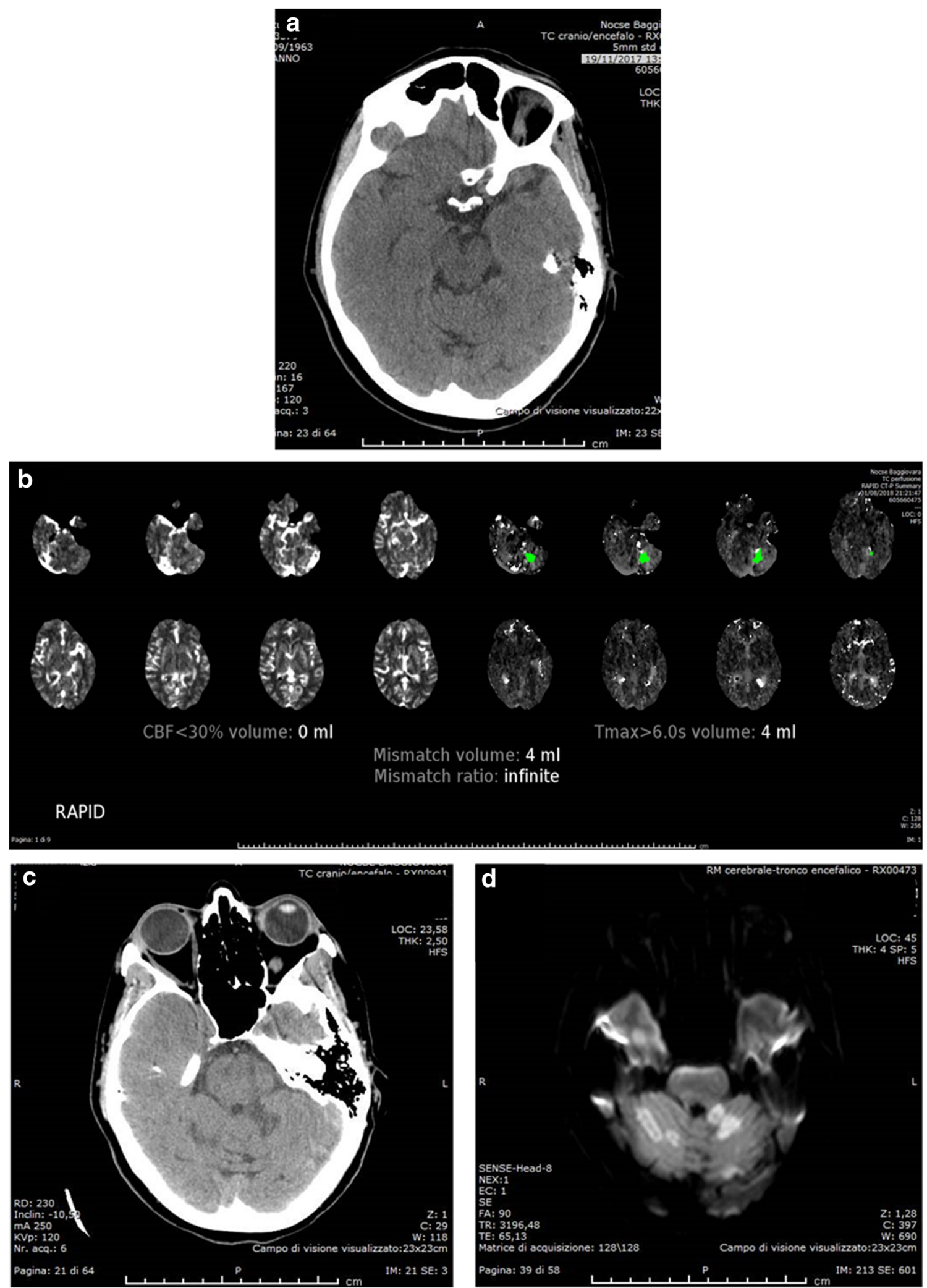

Fig. 4 Left small cerebellar ischaemic lesion visible on initial NCCT (a) as hypodensity, not recognised as ischaemic core by the automatic RAPID mismatch map (b) but confirmed on follow-up CT (c) and DWI (d) 
Acknowledgements Open access funding provided by Università degli Studi della Campania Luigi Vanvitelli within the CRUI-CARE Agreement.

Funding information This study and the use of RAPID software were supported by the Italian Ministry of Health, Young Researcher Grant-Ricerca Finalizzata Giovani Ricercatori 2013 (grant number GR-2013-02357885), title "Endovascular Therapy in acute ischaemic stroke: an observational prospective study of an interprovincial Hub and Spoke Model".

Data availability The data that support the findings of this study are available on request from the corresponding author [R.C.].

\section{Compliance with ethical standards}

Conflict of interest S.V. declares a conflict of interest: he has received speaker fees from Cerenovus.

A.Z. declares a conflict of interest: he has received speaker fees and consulting fees from Boehringer-Ingelheim, Medtronic, Cerenovus and advisory board from Daiichi Sankyo and Boehringer-Ingelheim and Stryker.

R.C., N.S., L.V., G.Z., F.S., G.B., L.P., F.C. declare that they have no conflict of interest.

Ethical approval All procedures performed in studies involving human participants were in accordance with the ethical standards of the institutional and/or national research committee and with the 1964 Helsinki declaration and its later amendments or comparable ethical standards. The study was approved by the local ethical committee (Area Vasta Emilia Nord).

Informed consent Informed consent was obtained from all individual participants included in the institutional stroke registries and in the study GR-2013-02357885.

Consent for publication Not applicable.

Code availability Not applicable.

Open Access This article is licensed under a Creative Commons Attribution 4.0 International License, which permits use, sharing, adaptation, distribution and reproduction in any medium or format, as long as you give appropriate credit to the original author(s) and the source, provide a link to the Creative Commons licence, and indicate if changes were made. The images or other third party material in this article are included in the article's Creative Commons licence, unless indicated otherwise in a credit line to the material. If material is not included in the article's Creative Commons licence and your intended use is not permitted by statutory regulation or exceeds the permitted use, you will need to obtain permission directly from the copyright holder. To view a copy of this licence, visit http://creativecommons.org/licenses/by/4.0/.

\section{References}

1. Nouh A, Remke J, Ruland S (2014) Ischemic posterior circulation stroke: a review of anatomy, clinical presentations, diagnosis, and current management. Front Neurol 5:30. https://doi.org/10.3389/ fneur.2014.00030 eCollection 2014

2. Merwick Á, Werring D (2014) Posterior circulation ischaemic stroke. BMJ 348:g3175. https://doi.org/10.1136/bmj.g3175

3. van der Hoeven EJ, Dankbaar JW, Algra A, Vos JA, Niesten JM, van Seeters T, van der Schaaf IC, Schonewille WJ, Kappelle LJ, Velthuis BK, DUST Investigators (2015) Additional diagnostic value of computed tomography perfusion for detection of acute ischemic stroke in the posterior circulation. Stroke 46(4):11131115. https://doi.org/10.1161/STROKEAHA.115.008718

4. Pallesen LP, Lambrou D, Eskandari A, Barlinn J, Barlinn K, Reichmann H, Dunet V, Maeder P, Puetz V, Michel P (2018) Perfusion computed tomography in posterior circulation stroke: predictors and prognostic implications of focal hypoperfusion. Eur J Neurol May 25(5):725-731. https://doi.org/10.1111/ene.13578

5. Bollwein C, Plate A, Sommer WH, Thierfelder KM, Janssen H, Reiser MF, Straube A, von Baumgarten L (2016) Diagnostic accuracy of whole-brain $\mathrm{CT}$ perfusion in the detection of acute infratentorial infarctions. Neuroradiology 58(11):1077-1085

6. Sparaco M, Ciolli L, Zini A (2019) Posterior circulation ischaemic strokea review part I: anatomy, aetiology and clinical presentations. Neurol Sci 40(10):1995-2006. https://doi.org/10.1007/s10072-019-03977-2

7. Sparaco M, Ciolli L, Zini A (2019) Posterior circulation ischemic stroke-a review part II: imaging and acute treatment. Neurol Sci 40(10):2007-2015. https://doi.org/10.1007/s10072-019-03936-x

8. Helseth EK, Lutsep HL (2018) Posterior cerebral artery stroke. Updated: Jul 30, 2018. https://emedicine.medscape.com/article/ 2128100-overview

9. Siniscalchi A, Sztajzel R, Malferrari G, Gallelli L (2017) The National Institutes of Health Stroke Scale: its role in patients with posterior circulation stroke. Hosp Top 95(4):79-81. https://doi.org/ 10.1080/00185868.2017.1322888

10. Pezzella FR, Pozzessere C, Siniscalchi A, Gallelli L, Anticoli S (2013) The cloud stroke unit: 24-hour acute stroke expertise-on-demand. Hosp Top 91(4):81-86. https://doi.org/10.1080/00185868.2013.848160

11. Meyer BC, Hemmen TM, Jackson CM, Lyden PD (2002) Modified National Institutes of Health Stroke Scale for use in stroke clinical trials: prospective reliability and validity. Stroke 33(5):1261-1266

12. Ukmar M, Degrassi F, Pozzi Mucelli RA, Neri F, Mucelli FP, Cova MA (2017) Perfusion CT in acute stroke: effectiveness of automatically-generated colour maps. Br J Radiol 90(1072): 20150472. https://doi.org/10.1259/bjr.20150472

13. Koenig M, Klotz E, Luka B, Venderink DJ, Spittler JF, Heuser L (1998) Perfusion CT of the brain: diagnostic approach for early detection of ischemic stroke. Radiology 209(1):85-93

14. Nabavi DG, Cenic A, Craen RA, Gelb AW, Bennett JD, Kozak R, Lee TY (1999) CT assessment of cerebral perfusion: experimental validation and initial clinical experience. Radiology 213(1):141-149

15. Cianfoni A, Colosimo C, Basile M, Wintermark M, Bonomo L (2007) Brain perfusion CT: principles, technique and clinical applications. Radiol Med 112(8):1225-1243

16. Böck JC, Henrikson O, Götze AH, Wlodarczyk W, Sander B, Felix R (1995) Magnetic resonance perfusion imaging with gadoliniumDTPA. A quantitative approach for the kinetic analysis of first-pass residue curves. Investig Radiol 30(12):693-699

17. Lee IH, You JH, Lee JY, Whang K, Kim MS, Kim YJ, Lee MS, Brain Research Group (2010) Accuracy of the detection of infratentorial stroke lesions using perfusion CT: an experimenterblinded study. Neuroradiology 52(12):1095-1100. https://doi.org/ 10.1007/s00234-010-0689-2

18. Campbell BC, Christensen S, Levi CR, Desmond PM, Donnan GA, Davis SM, Parsons MW (2012) Comparison of computed tomography perfusion and magnetic resonance imaging perfusiondiffusion mismatch in ischemic stroke. Stroke 43(10):2648-2653

19. Wintermark M, Flanders AE, Velthuis B, Meuli R, van Leeuwen M, Goldsher D, Pineda C, Serena J, van der Schaaf I, Waaijer A, Anderson J, Nesbit G, Gabriely I, Medina V, Quiles A, Pohlman S, Quist M, Schnyder P, Bogousslavsky J, Dillon WP, Pedraza S (2006) Perfusion-CT assessment of infarct core and penumbra: receiver operating characteristic curve analysis in 130 patients suspected of acute hemispheric stroke. Stroke 37(4):979-985

20. Lui YW, Tang ER, Allmendinger AM, Spektor V (2010) Evaluation of CT perfusion in the setting of cerebral ischemia: 
patterns and pitfalls. AJNR Am J Neuroradiol 31(9):1552-1563. https://doi.org/10.3174/ajnr.A2026

21. d'Esterre CD, Roversi G, Padroni M, Bernardoni A, Tamborino C, De Vito A, Azzini C, Marcello O, Saletti A, Ceruti S, Lee TY, Fainardi E (2015) CT perfusion cerebral blood volume does not always predict infarct core in acute ischemic stroke. Neurol Sci 36(10):1777-1783. https://doi.org/10.1007/s10072-015-2244-8

22. Campbell BC, Christensen S, Levi CR, Desmond PM, Donnan GA, Davis SM, Parsons MW (2011) Cerebral blood flow is the optimal CT perfusion parameter for assessing infarct core. Stroke 42(12): 3435-3440. https://doi.org/10.1161/STROKEAHA.111.618355

23. Dehkharghani S, Bammer R, Straka M, Albin LS, Kass-Hout O, Allen JW, Rangaraju S, Qiu D, Winningham MJ, Nahab F (2015) Performance and predictive value of a user-independent platform for CT perfusion analysis: threshold-derived automated systems outperform examiner-driven approaches in outcome prediction of acute ischemic stroke. AJNR Am J Neuroradiol 36(8):1419-1425. https://doi.org/10.3174/ajnr.A4363

24. de Lucas EM, Sanchez E, Gutierrez A et al (2008) CT protocol for acute stroke: tips and tricks for general radiologists. Radiographics 28:1673-1687

25. Wintermark M, Albers GW, Alexandrov AV, Alger JR, Bammer R, Baron JC, Davis S, Demaerschalk BM, Derdeyn CP, Donnan GA, Eastwood JD, Fiebach JB, Fisher M, Furie KL, Goldmakher GV, Hacke W, Kidwell CS, Kloska SP, Köhrmann M, Koroshetz W, Lee TY, Lees KR, Lev MH, Liebeskind DS, Ostergaard L, Powers WJ, Provenzale J, Schellinger P, Silbergleit R, Sorensen AG, Wardlaw J, Wu O, Warach S (2008) Acute stroke imaging research roadmap. Stroke 39:1621-1628

26. Eastwood JD, Lev MH, Azhari T, Lee TY, Barboriak DP, Delong DM, Fitzek C, Herzau M, Wintermark M, Meuli R, Brazier D, Provenzale JM (2002) CT perfusion scanning with deconvolution analysis: pilot study in patients with acute middle cerebral artery stroke. Radiology 222:227-236

27. Miles KA, Griffiths MR (2003) Perfusion CT: a worthwhile enhancement? Br J Radiol 76:220-231

28. Lansberg MG, Straka M, Kemp S, Mlynash M, Wechsler LR, Jovin TG, Wilder MJ, Lutsep HL, Czartoski TJ, Bernstein RA, Chang CW, Warach S, Fazekas F, Inoue M, Tipirneni A, Hamilton SA, Zaharchuk G, Marks MP, Bammer R, Albers GW, DEFUSE 2 study investigators (2012) MRI profile and response to endovascular reperfusion after stroke (DEFUSE 2): a prospective cohort study. Lancet Neurol 11: 860-867. https://doi.org/10.1016/S1474-4422(12)70203-X3

29. Olivot J-M, Mlynash M, Thijs VN, Kemp S, Lansberg MG, Wechsler L, Bammer R, Marks MP, Albers GW (2009) Optimal Tmax threshold for predicting penumbral tissue in acute stroke. Stroke 40:469-475. https://doi.org/10.1161/STROKEAHA.108.526954

30. Wheeler HM, Mlynash M, Inoue M, Tipirneni A, Liggins J, Zaharchuk G et al (2013) Early diffusion-weighted imaging and perfusion-weighted imaging lesion volumes forecast final infarct size in DEFUSE 2. Stroke 44:681-685. https://doi.org/10.1161/ STROKEAHA.111.0001355

31. Shih LC, Saver JL, Alger JR, Starkman S, Leary MC, Vinuela F, Duckwiler G, Gobin YP, Jahan R, Villablanca JP, Vespa PM, Kidwell CS (2003) Perfusion-weighted magnetic resonance imaging thresholds identifying core, irreversibly infarcted tissue. Stroke 34: 1425-1430. https://doi.org/10.1161/01.STR.0000072998.70087.E9

32. Kidwell CS, Jahan R, Gornbein J, Alger JR, Nenov V, Ajani Z, Feng L, Meyer BC, Olson S, Schwamm LH, Yoo AJ, Marshall RS, Meyers PM, Yavagal DR, Wintermark M, Guzy J, Starkman S, Saver JL, MR RESCUE Investigators (2013) A trial of imaging selection and endovascular treatment for ischemic stroke. N Engl J Med 368:914-923. https://doi.org/10.1056/NEJMoa1212793

33. Mokin M, Levy EI, Saver JL, Siddiqui AH, Goyal M, Bonafé A, Cognard C, Jahan R, Albers GW, SWIFT PRIME Investigators (2017) Predictive value of RAPID assessed perfusion thresholds on final infarct volume in SWIFT PRIME (Solitaire With the Intention for Thrombectomy as Primary Endovascular Treatment). Stroke 48(4): 932-938. https://doi.org/10.1161/STROKEAHA.116.015472

34. Kudo K, Sasaki M, Yamada K, Momoshima S, Utsunomiya H, Shirato H, Ogasawara K (2010) Differences in CT perfusion maps generated by different commercial software: quantitative analysis by using identical source data of acute stroke patients. Radiology 254(1):200-209. https://doi.org/10.1148/radiol.254082000

35. Campbell BC, Yassi N, Ma H, Sharma G, Salinas S, Churilov L, Meretoja A, Parsons MW, Desmond PM, Lansberg MG, Donnan GA, Davis SM (2015) Imaging selection in ischemic stroke: feasibility of automated CT-perfusion analysis. Int J Stroke 10(1):51-54. https://doi.org/10.1111/ijs.12381

36. Vyas D, Bohra V, Karan V, Huded V (2019) Rapid processing of perfusion and diffusion for ischemic strokes in the extended time window: an Indian experience. Ann Indian Acad Neurol 22(1):9699. https://doi.org/10.4103/aian.AIAN.142.18

37. Straka M, Albers GW, Bammer R (2010) Real-time diffusion-perfusion mismatch analysis in acute stroke. J Magn Reson Imaging 32:1024-1037

38. Pallesen LP, Gerber J, Dzialowski I, van der Hoeven EJ, Michel P, Pfefferkorn T, Ozdoba C, Kappelle LJ, Wiedemann B, Khomenko A, Algra A, Hill MD, von Kummer R, Demchuk AM, Schonewille WJ, Puetz V, BASICS Study Group (2015) Diagnostic and prognostic impact of pc-ASPECTS applied to perfusion CT in the basilar artery international cooperation study. J Neuroimaging 25(3): 384-389. https://doi.org/10.1111/jon.12130

39. Sporns P, Schmidt R, Minnerup J, Dziewas R, Kemmling A, Dittrich R, Zoubi T, Heermann P, Cnyrim C, Schwindt W, Heindel W, Niederstadt T, Hanning U (2016) Computed tomography perfusion improves diagnostic accuracy in acute posterior circulation stroke. Cerebrovasc Dis 41(5-6):242-247. https://doi.org/10.1159/000443618

40. Sharon et al (2016) The predictive value of a targeted posterior fossa multimodal stroke protocol for the diagnosis of acute posterior ischemic stroke. Neurovasc Imaging 2:3. https://doi.org/10.1186/ s40809-016-0013-6

41. Bamford J, Sandercock P, Dennis M, Burn J, Warlow C (1991) Classification and natural history of clinically identifiable subtypes of cerebral infarction. Lancet 337(8756):1521-1526

42. Pexman JH, Barber PA, Hill MD, Sevick RJ, Demchuk AM, Hudon ME, Hu WY, Buchan AM (2001) Use of the Alberta stroke program early CT score (ASPECTS) for assessing CT scans in patients with acute stroke. AJNR Am J Neuroradiol 22:1534-1542

43. Puetz V, Dzialowski I, Hill MD, Demchuk AM (2009) The Alberta stroke program early $\mathrm{CT}$ score in clinical practice: what have we learned? Int J Stroke 4:354-364

44. Aviv RI, Mandelcorn J, Chakraborty S, Gladstone D, Malham S, Tomlinson G, Fox AJ, Symons S (2007) Alberta stroke program early CT scoring of CT perfusion in early stroke visualization and assessment. AJNR Am J Neuroradiol 28:1975-1980

45. Alemseged F, Shah DG, Bivard A, Kleinig TJ, Yassi N, Diomedi M, Di Giuliano F, Sharma G, Drew R, Yan B, Dowling RJ, Bush S, Sallustio F, Caltagirone C, Mercuri NB, Floris R, Parsons MW, Levi CR, Mitchell PJ, Davis SM, Campbell BC (2019) Cerebral blood volume lesion extent predicts functional outcome in patients with vertebral and basilar artery occlusion. Int J Stroke 14(5):540 547. https://doi.org/10.1177/1747493017744465

46. Kauw F, Heit JJ, Martin BW, van Ommen F, Kappelle LJ, Velthuis BK, de Jong HWAM, Dankbaar JW, Wintermark M (2019) Computed tomography perfusion data for acute ischemic stroke evaluation using Rapid Software: pitfalls of automated postprocessing. J Comput Assist Tomogr. doi: https://doi.org/10.1097/RCT.0000000000000946.

Publisher's note Springer Nature remains neutral with regard to jurisdictional claims in published maps and institutional affiliations. 